\title{
Features of the Microstructure Development under Conditions, Reproducing the Process of Friction Stir Welding. Molecular-Dynamics Study
}

\author{
Anton Yu. Nikonov ${ }^{1,2, a)}$, Andrey I. Dmitriev ${ }^{1,2, ~ b)}$, Evgeniy A. Kolubaev ${ }^{2,3, c)}$, \\ and Valeriy E. Rubtsov $2,3, \mathrm{~d})$ \\ ${ }^{1}$ National Research Tomsk State University, Tomsk, 634050, Russia \\ ${ }^{2}$ Institute of Strength Physics and Materials Science SB RAS, Tomsk, 634055, Russia \\ ${ }^{3}$ National Research Tomsk Polytechnic University, Tomsk, 634050, Russia \\ a) Corresponding author: anickonoff@ispms.tsc.ru \\ b) dmitr@ispms.tsc.ru \\ c) eak@ispms.tsc.ru \\ d) rvy@ispms.tsc.ru
}

\begin{abstract}
Friction stir welding is a recently developed technology which is used in various branches of modern engineering. The basis of this technology is the friction of the rotating cylindrical or specially shaped tool between two metal plates brought together either to meet their ends of one above another with the overlap. When applying the FSW process in various economical sectors, the important task is to study the mechanisms and identify the physical laws and factors leading to formation of structural inhomogeneities and discontinuities in the weld seam. This paper analyzes the basic mechanisms behind the structural state generation in the material subjected to severe plastic deformation and heating. To investigate the atomic mechanisms of structural changes in FSW, the modeling at atomic scale has been carried out. Results of work can be a basis for new knowledge about the microstructure evolution in FSW.
\end{abstract}

Keywords: microstructure, deformation, mechanical activation, molecular dynamics

\section{INTRODUCTION}

Friction stir welding (FSW) is a relatively new method of obtaining non-detachable joints of materials (patented by The Welding Institute in UK (TWI), (UK) in 1991 [1]). Recent year studies have shown that FSW is an effective way to obtain high quality joints for structures of various dimensions and shapes, including sheets, 3D profile structures, and pipes. Also it was used to restore the worn parts or to modify and improve the microstructure of materials, for healing cracks and casting flaws. Possessing the broad technological capabilities for obtaining permanent joints of details or units, it can be used as an alternative to riveted joints, electric arc welding, electron beam and laser welding as well as for welding the dissimilar materials. Thus, FSW becomes a versatile technology that has a great potential in various industries.

FSW is one example of the practical use of the acceleration effect of diffusion processes due to mechanical activation. Since mechanoactivated processes are inseparably linked with the intensive formation of discontinuities, the generation of structural defects of different levels, the mass transfer, the most preferable one is to use methods of discrete description of the simulated environment. The aim of this work is to analyze the basic regularities and mechanisms of structural state formation in the material subjected at atomic scale to severe plastic deformation and elevated temperatures.

International Conference on Physical Mesomechanics of Multilevel Systems 2014

AIP Conf. Proc. 1623, 439-442 (2014); doi: 10.1063/1.4898976

(C) 2014 AIP Publishing LLC 978-0-7354-1260-6/\$30.00 


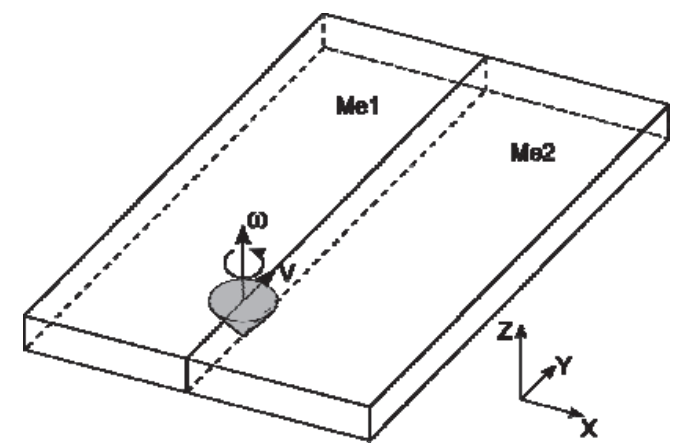

FIGURE 1. Schematic representation of the simulated sample

\section{NUMERICAL MODEL OF THE PROCESS}

In order to study the implementation of possible atomic mechanisms in the loading conditions identical to those used in FSW, we carried out modeling the FSW by a rotating tool in the form of rigid cone that moves along the boundary between two grains. Schematic representation of the simulated sample is shown in Fig. 1. The study was conducted in the framework of molecular dynamics method using the software package LAMMPS [2-4]. The interaction between atoms is described within the embedded atom method [3,4]. Modeled sample was considered as NVE ensemble that maintains the number of particles $N$, the occupied volume $V$ and the energy of the system $E$. Velocity-Verlet integrator was used. The equations of motion were integrated with a time step $\Delta t=0.001 \mathrm{ps}$.

The two metal crystallites' sizes (Me1 and Me2) were $10.8 \times 21.7 \times 3.6 \mathrm{~nm}$. The part of atoms shaped in cone was simulating a tool rotating at constant angular velocity $\omega$. The height and a base diameter of the tool were $3.6 \mathrm{~nm}$. Also the cone was moved along the boundary at feeding rate $V$. Additionally an oscillation of the tool with amplitude A and frequency $\omega^{\prime}$ was modeled.

\section{SIMULATION RESULTS}

Originally we modeled the process which reproduced at the atomic scale the loading conditions used in FSW of two ideal copper crystallites. The rotation rate and feeding rate of the tool were chosen $V=50 \mathrm{~m} / \mathrm{s}$ and $\omega=0.1 \mathrm{ps}^{-1}$ respectively. Initially simulation was performed without oscillation of the tool. Heat removal from the sample was realized by using of additional layers with artificial viscosity. Periodic boundary conditions were set in directions along to axis $X$ and $Y$ to account for extended sizes of the simulated sample.

Analysis of the structure of the sample showed the intermixing and stirring of dissimilar atoms as a result the FSW tool pass at the inter-crystallite boundary. Figure 2(a) shows the mapping of atoms on the plane XOY for a moment of time corresponding to the working tool pass along the entire length of the boundary between two metals. Differently shaded are the gray atoms originally belonged to either Mel or Me 2 crystallites. It is seen that the trace of passing instrument consists of two parts. The area with the crystal lattice irregularities has been found directly behind the tool. Atoms of Me1 and Me2 fragments present in it in approximately equal proportions. The thickness of this area is comparable with the size of the rotating tool. When tool passes far along the boundary at a distance comparable with its diameter, there a region where atoms start occupying the original position of the crystal lattice due to relaxation in the nodes formed. The specificity of this area is the gradient distribution of atoms belonging to grains Me1 and Me2. Figure 3(a) shows the projection of the central fragment atoms on the plane XOZ. It can be seen that the width of the layer where a mixing of atoms is considerably wider in the upper part of the sample. This is caused by the conical shape of the tool. Analysis of the crystal lattice structure by using the atomic bond local topology search algorithm [5] revealed the structural defects. However, their volume fraction relative to the total number of atoms is negligible.

By setting the oscillation of tool along the axis of rotation additional to the rotational and translational motion, we can generate a number of structural defects such as nanopores. Similar results were obtained in modeling and experiment by increasing feed rate [6]. We can assume that it is possible to avoid the formation of pores by reducing the feeding rate of the tool. 


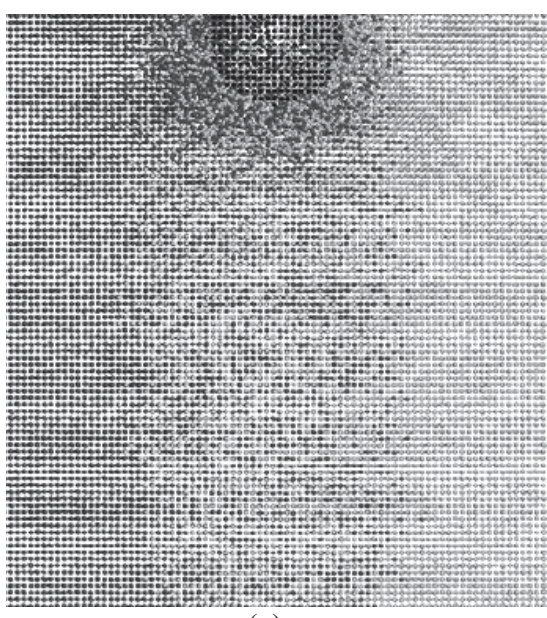

(a)

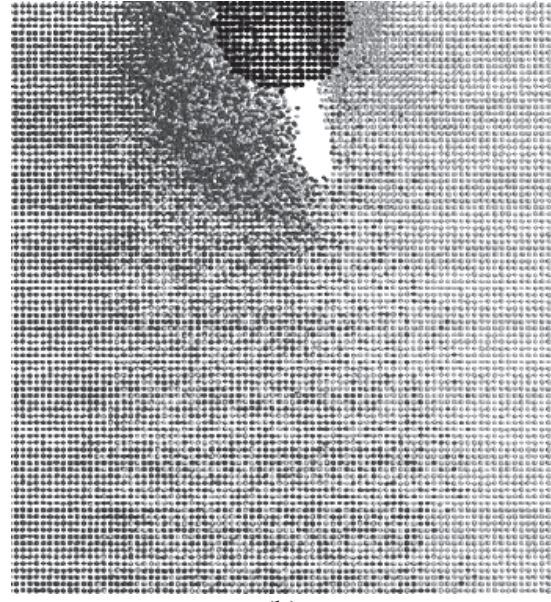

(b)

FIGURE 2. Projection of atoms on the plane $X O Y$ after the passage tool in conjugate pair $\mathrm{Cu}-\mathrm{Cu}$ without oscillatory motion of the tool (a), with oscillation (b)

The results showed that the final atomic lattice structure in the weld formed after passing the tool also corresponds to fcc lattice. A distinctive feature of this "trace" is a large number of structural defects (vacancies, nanopores, dislocations, and stacking faults). The event of generation of a nanopore is clearly visible in the figure below. Another result of the oscillatory motion of the tool is the same width throughout the depth of the sample of the area where there is a mixing of atoms, originally belonging to different crystallites (comp. Fig. 3(a, b)).

At the next stage of the research we reproduced on the atomic scale the loading conditions used in FSW of dissimilar material crystallites such as $\mathrm{Cu}(\mathrm{Me} 1)$ and $\alpha-\mathrm{Fe}(\mathrm{Me} 2)$. Along the $\mathrm{Y}$ direction periodic conditions were simulated. In the $X$ direction the hard boundary conditions were set up. Sizes of simulated fragments of Me1 and Me 2 were equal to $21.7 \times 21.7 \times 3.5 \mathrm{~nm}$. Initial crystallographic orientation of both crystallites was chosen as [100] [010] and [001] along the respective axes. Tool speed was $V=50 \mathrm{~m} / \mathrm{s}$ and $\omega=0.1 \mathrm{ps}^{-1}$. Additionally oscillatory motion of the tool was modelled. As before the removal of heat from the specimen is realized by introducing an artificial viscosity of atoms belonging to the two buffer layers peripheral with respect to the plane of coupling of both crystallites.

Simulation results showed that after the passage of the rotating tool along the boundary, we can see there a complex configuration comprising the intermix of copper and iron atoms. The detailed analysis of the resultant structure showed some $\mathrm{Cu}$-atoms substituted $\mathrm{Fe}$ ones in the $\alpha$-Fe bcc lattice in the vicinity of initial joint interface and vice versa iron atoms substituted copper ones in the fcc lattice.

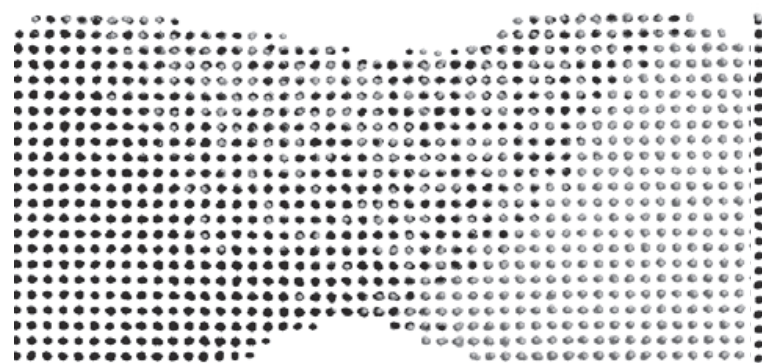

(a)

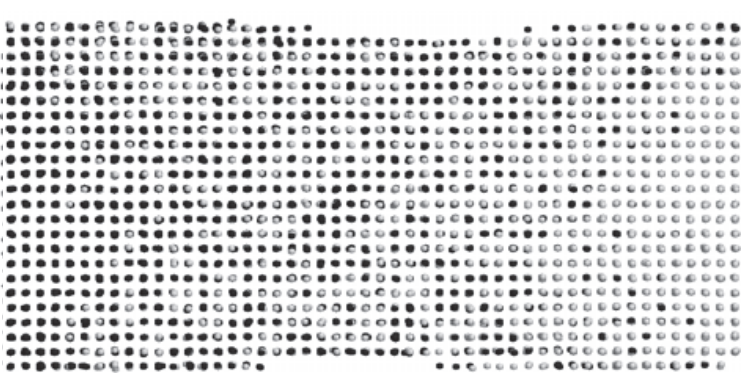

(b)

FIGURE 3. Projection of atoms of structure fragment near "trace" on the plane $X O Z$ after the passage tool without oscillatory motion of the tool (a), with oscillation (b) 


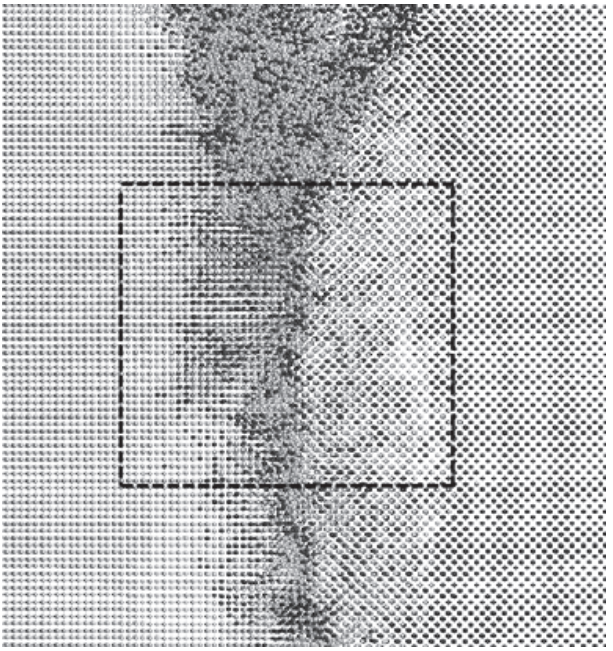

(a)

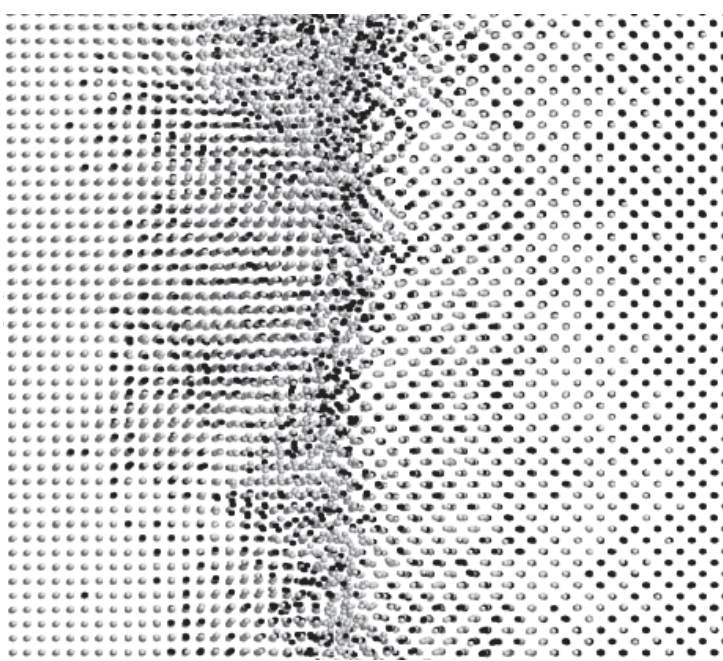

(b)

FIGURE 4. Projection of atoms on the plane $X O Y$ after the passage tool in conjugate pair $\mathrm{Cu}-\alpha$-Fe. Image (b) corresponds to the fragment framed in (a)

As previously, the gradient character of atom substitution zone in both metals has been observed, but the width of this zone is less in comparison with the results obtained for the $\mathrm{Cu} / \mathrm{Cu}$ interface. This can be explained by the differences in crystal lattice structures. This also explains the presence of numerous structural defects along the boundary obtained after passing the instrument.

\section{CONCLUSIONS}

On the basis of the research results the conclusions can be made as follows. In spite of the fundamental differences in characteristic spatial and temporal parameters, computer simulation results are in good qualitative agreement with those of experimental studies. Computer model may be a test bed serving for better understanding the basic laws of structural inhomogeneitiy origin in FSW. The simulation results obtained can be those of practical importance. They allow discovering new ways and mechanisms to obtain non-equilibrium states in the crystal lattice due to the initiation of the mechanically activated metal interdiffusion at the atomic level.

\section{ACKNOWLEDGMENTS}

Work is sponsored by the Ministry of Education and Science of the Russian Federation (contract No. 02.G25.31.0063) as part of the Government Resolutions of the Russian Federation No. 218, partly by the Program for Basic Scientific Research of the State Academy of Science on 2013-2020 and RFBR grants Nos. 1408-31662 and 14-08-91330.

\section{REFERENCES}

1. W. M. Thomas, E. D. Nicholas, J. C. Needham, M. G. Murch, P. Templesmith, and C. J. Dawes, G.B. patent 9125978.8 (1991).

2. A. I. Dmitriev, A. Yu. Nikonov, and S. G. Psakhie, Phys. Mesomech. 14(1-2), 24 (2011).

3. A. I. Dmitriev and A. Y. Nikonov, Tech. Phys. Lett. 39(8), 709 (2013).

4. M. P. Bondar, S. G. Psakhie, A. I. Dmitriev, and A. Y. Nikonov, Phys. Mesomech. 16(3), 191 (2013).

5. D. Honeycutt J. and H. C. Andemen, J. Phys. Chem. 91, 4950 (1987).

6. P. A. Vityaz, V. E. Panin, A. V. Byeli, and A. V. Kolubaev, Mechanics of plastic deformation and fracture of surface-hardened solids in friction, Phys. Mesomech. 5(1), 15 (2002). 